\title{
EFFETS DES DIFFÉRENTES FRACTIONS DU GRADIENT DE PERCOLL DISCONTINU SUR LA MOBILITÉ DES SPERMATOZOIDES HUMAINS.
}

\author{
A. Saad*, J. F. Guérin**, J. C. Czyba**
}

* Laboratoire de biologie de la reproduction et de cytogénétique C.H.U. Farhat Hached, 4000 Sousse, TUNISIE

** Laboratoire de biologie de la reproduction et du développement Faculté de Médecine, 8, avenue Rockefeller, 69008 Lyon, FRANCE

EFFECTS OF VARIOUS FRACTIONS OF DISCONTINUED PERCOLL GRADIENT ON THE MOBILITY OF HUMAN SPERMATOZOA : The centrifugation on discontinued Percoll gradient is a technique of sperm preparation, which has enabled better success of in-vitro fertilization. In this work, we have analysed the movements of spermatozoa separated in different Percoll fractions after 4 hours of incubation at $37^{\circ} \mathrm{C}$ and under $5 \%$ of $\mathrm{CO}_{2}$. These measures are made by computer assisted videomicrography (Cell Soft). From this study, we notice that the more the Percoll concentration increases, the greater is the curvilinear velocity, the linearity and the lateral amplitude of the head. The $100 \%$ and $90 \%$ Percoll fractions help to get spermatozoa with good movement features, indicating the use of this technique in medically assisted procreation. Key-words : Human sperm, Percoll, motility, videomicrography. Andrologie, 1991, 1 : 9-10

Différentes techniques sont utilisées pour la préparation du sperme pour les procréations médicalement assistées. Parmi elles, la migration ascendante est utilisée en routine par de nombreux laboratoires de biologie de la reproduction. Cependant, cette technique ne permet pas d'obtenir un nombre suffisant de spermatozoïdes lorsque l'oligoasthénospermie est importante. La centrifugation sur gradient de Percoll discontinu a permis d'améliorer les succès de la fécondation in-vitro (3). Cette technique est actuellement utilisée surtout en cas d'asthénozoospermie ou de leucospermie importante. Elle permet d'obtenir une bonne concentration en spermatozoides à morphølogie normale et de bonne mobilité.

Parmi les méthodes d'analyse de la mobilité, la vidéomicrographie assistée par ordinateur permet une description objective et fine des mouvements des spermatozoides. Dans ce travail, nous analysons à l'aide de cetce méthode, les mouvements des spermatozoildes humains séparés dans différentes couches de Percoll et incubés à $37^{\circ} \mathrm{C}$ sous $5 \%$ de $\mathrm{C} 02$ pendant 4 heures.

\section{MATERIEL ET METHODES}

Nous avons analysé 15 spermes provenant de 15 donneurs volontaires, en bonne santé, supposés fertiles. Les prélèvements sont faits par masturbation, dans un flacon stérile, au laboratoire. Chaque échantillon est caractérisé par un volume de 1,5 à $3,5 \mathrm{ml}$, une concentration en spermatozoîdes de 50 à $100 \times 10^{6} / \mathrm{ml}$, une mobilité $\geq 50 \%$ et une tératozoospermie $\leq 40 \%$.

La séparation des spermatozoïdes est réalisée selon la méthode simplifiée de la technique décrite par BERGER et al. (1985) (2). Une solution isotonique de Percoll est obtenue en mélangeant 9 parts de Percoll (Sigma Chemical Co., St-Louis, M.O.) et 1 part de milieu de Eagle (x 10) (Biomérieux, Choubonnières-lesBains, France). Cette solution constitue la fraction $100 \%$ (P 100). Elle est diluée avec du milieu $\mathrm{B}_{2}$ de Menezo pour obtenir les fractions $90,80,70$, 60 et $40 \%$ qui sont toutes isotoniques. Le gradient discontinu est réalisé dans des tubes en pipettant délicatement $1 \mathrm{ml} \mathrm{de} 100,90,80,70$ et 60 et finalement $1,5 \mathrm{ml}$ de $40 \%$ de façon que les interfaces soient visibles entre les couches. $1 \mathrm{ml}$ de sperme est alors déposé à la surface du gradient. Chaque gradient est centrifugé pendant $20 \mathrm{mn}$ à $330 \mathrm{~g}$. Le plasma séminal et la fraction $40 \%$ sont éliminés. Les fractions 60 (P 60), 70 (P 70), 80 (P 80), 90 (P 90) et 100\% (P 100) sont séparées et déposées chacune dans un tube conique (Nunc, 34 7880, Denmark). Chaque fraction est par la suite diluée dans deux v̀olumes de $B_{2}$ puis centrifugée pendant $10 \mathrm{mn}$ à $400 \mathrm{~g}$. Chaque culot est resuspendu dans $0,5 \mathrm{ml} \mathrm{de} \mathrm{B}_{2}$ et incubé à $37^{\circ} \mathrm{C}$ sous $5 \%$ de $\mathrm{CO}_{2}$ pendant 4 heures. L'analyse des mouvements des spermatozoïdes est réalisée pour chaque fraction à l'aide de la vidéomicrographie assistée par ordinateur (système Cell Soft Cryo Resources Inc., New York, N.Y.). Les mesures sont réalisées à $37^{\circ} \mathrm{C}$ utilisant les chambres de Makler de $10 \mu \mathrm{m}$ de profondeur. Les paramètres mesurés sont

- la vitesse curvilinéaire moyenne (VCL)

- l'amplitude maximale de déplacement latéral de la tête $\left(\mathrm{ALH}_{\max }\right)$

- ta linéarité moyenne (LIN)

- la fréquence du changement de direction de la tête par rapport à l'axe de déplacement (BCF).

L'étude de l'influence des gradients de Percoll sur les mouvements des spermatozoides est réalisée par analyse de variance.

\section{RESULTATS}

L'analyse de variance a permis de déceler un effet hautement significatif des différentes fractions de Percoll sur $\mathrm{ALH}_{\max }(\mathrm{F}=17,99$; $\mathrm{p}<0,001) . \mathrm{ALH}_{\max }$ est la plus élevée chez les spermatozoïdes récupérés dans les fractions $100 \%$ et $90 \%$ par rapport à ceux récupérés dans les autres fractions (fig. 1, tableau 1): $\mathrm{P}_{100}=\mathrm{P}_{90}>\mathrm{P}_{80}>\mathrm{P}_{70}>\mathrm{P}_{60}(\mathrm{P}<0,05)$.

$\mathrm{La}$ vitesse curvilinéaire varie significativement d'une fraction Percoll à une autre $(F=32,59$; $\mathrm{P}<0,001)$. Elle varie dans le même sens que $\mathrm{ALH}_{\text {max }}$; elle est maximale chez les spermatozoídes récupérés dans les fractions $100 \%$ et $90 \%$ et diminue avec la concentration en Percoll (fig. 2, tableau 1):

$\mathrm{P}_{100}=\mathrm{P}_{90}>\mathrm{P}_{80}>\mathrm{P}_{70}>\mathrm{P}_{60}(\mathrm{P}<0,05)$.

La linéarité varie aussi en fonction des fractions de Percoll ( $F=8,29 ; \mathrm{P}<0,001)$. Une concentration très élevée en Percoll $\left(\mathrm{P}_{100}\right)$ permet d'avoir une trajectoire très linéaire. Cette trajectoire est la moins linéaire chez les spermatozoïdes récupérés dans les fractions de Percoll les plus concentrées (fig. 4, tableau 1) : $\mathrm{P}_{100}=\mathrm{P}_{90}>\mathrm{P}_{80}>\mathrm{P}_{70}>\mathrm{P}_{60}(\mathrm{P}<0,05)$.

Tableau 1.

Caractéristiques des mouvements des spermatozoïdes séparés dans différentes fractions de Percoll (les valeurs sont exprimées en moyenne \pm S.E.M.)

\begin{tabular}{|c|c|c|c|c|c|}
\hline \multirow{2}{*}{$\begin{array}{r}\text { Percoll } \\
\text { Mouvements }\end{array}$} & \multirow[b]{2}{*}{$\mathrm{P} 100$} & \multirow[b]{2}{*}{ P90 } & \multirow[b]{2}{*}{ P80 } & \multirow[b]{2}{*}{$\mathrm{P} 70$} & \multirow[b]{2}{*}{ P60 } \\
\hline & & & & & \\
\hline \multirow[t]{2}{*}{ 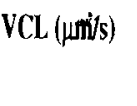 } & 82,39 & 86,63 & 78,93 & 72,29 & 66,53 \\
\hline & $\pm 2,00$ & $\pm 1,85$ & $\pm 2,45$ & $\pm 1,65$ & $\pm 2,30$ \\
\hline \multirow[t]{2}{*}{ ALHmax $(\mu \mathrm{m})$} & 3,27 & 3,65 & 3,58 & 3,51 & 3,58 \\
\hline & $\pm 0,10$ & $\pm 0,13$ & $\pm 0,12$ & $\pm 0,14$ & $\pm 0,13$ \\
\hline \multirow[t]{2}{*}{ LIN } & 67,80 & 72,9 & 67,7 & 64,5 & 59,8 \\
\hline & $\pm 2,60$ & $\pm 1,70$ & $\pm 2,50$ & $\pm 3,10$ & $\pm 2,10$ \\
\hline \multirow[t]{2}{*}{$\mathrm{BCF}(\mathrm{HZ})$} & 13,56 & 14,46 & 13,75 & 13,26 & 12,79 \\
\hline & $\pm 0,28$ & $\pm 0,15$ & $\pm 0,26$ & $\pm 0,27$ & $\pm 0,28$ \\
\hline
\end{tabular}




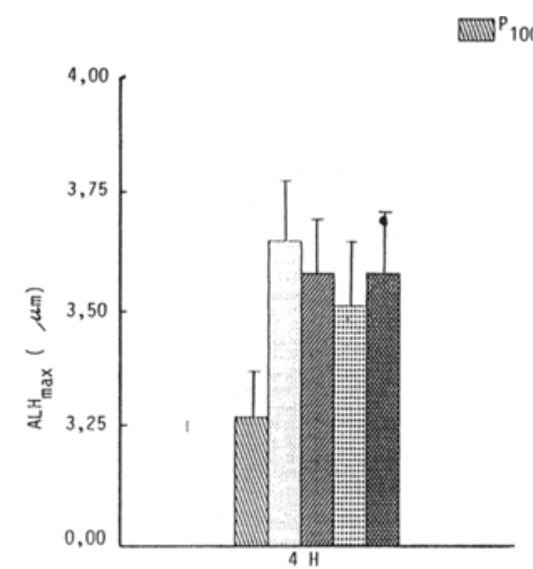

Figure 1 : ALH max en foncction des fractions de Percoll

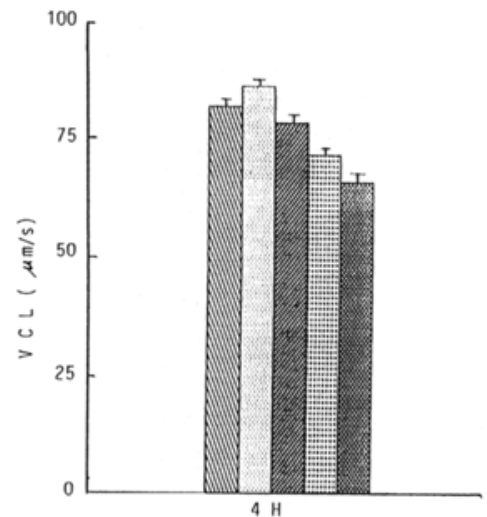

Figure 2: VCL en fonction des fractions de Percoll

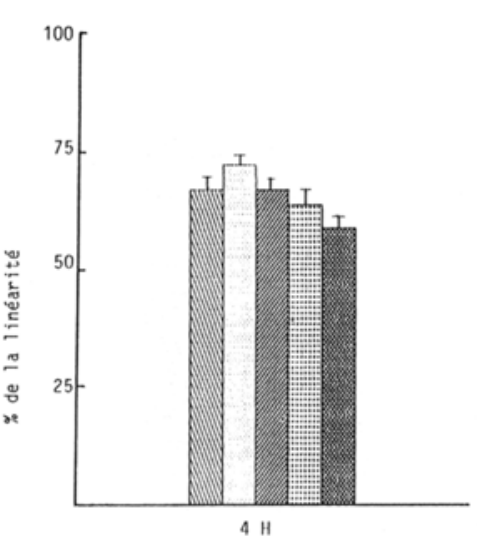

Figure 3 : LIN en fonction des fractions de Percoll

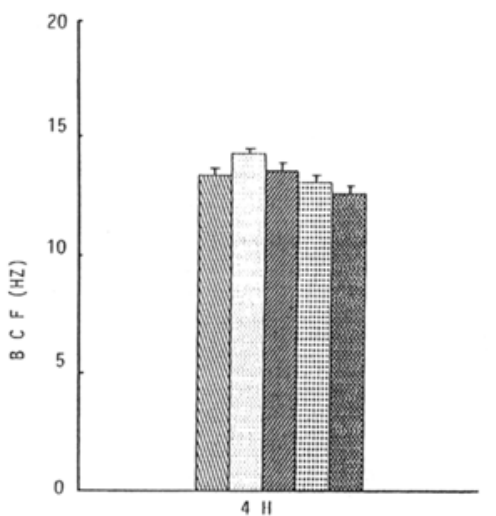

Figure 4: $\mathrm{BCF}$ en fonction des fractions de Percoll

\section{DISCUSSION}

La centrifugation sur gradient de Percoll discontinu est une technique qui améliore les capacités fécondantes des spermatozoïdes et leur capacité de pénétration dans les ovovytes de Hamster dépellucidés (6). Nos résultats montrent que la concentration en Percoll modifie les mouvements des spermatozoïdes : plus cette concentration augmente, plus la vitesse curvilinéaire, la linéarité et l'amplitude de déplacement latéral de lâ tête des spermatozoïdes augmentent. Plusieurs auteurs ont essayé d'expliquer les bases de cet effet du Percoll sur les spermatozoïdes. Le Percoll protège les spermatozoides contre une production excessive d'oxygène réactif qui serait à l'origine d'une perturbation de la fonction membranaire (1).

Certains auteurs pensent que le Percoll éliminerait certains facteurs inhihiteurs de la fécondation présents chez certains patients hypofertiles. D'autres auteurs ont montré que le Percoll permettrait d'obtenir des spermatozoides avec des anomalies chromatiniennes réduites par rapport à ceux récupérés par la technique de migration ascendante (5).
Nous pouvons conclure que les fractions $100 \%$ et $90 \%$ de Percoll permettent d'obtenir des spermatozoides avec de très bonnes caractéristiques des mouvements et une très bonne survie indiquant l'usage de cette technique en procréation médicalement assistée.

\section{REFERENCES}

1. - AITKEN R.J. Assessment of sperm function for I.V.F. Hum. Reprod. 1988 3: 89-95

2. - BERGER T.,MARRS R.P. MOYER D.L. Comparison of techniques for selection of motile spermatozoa. Fertil. Steril. 1985, 43: 268-273

3. - GUERIN J.F., MATHIEU C., LORNAGE J., PINATEL J.C., BOULIEU D. Improvement of survival and fertilizing capacity of human spermatozoa in an IVF programme by selection on discontinuous Percoll gradients. Human Reproduction, 1989 4: 798-804

4. - HYNE R.V., STOJANOFF A., CLARKE G.N., LOPARA A., JOHNSTON W.I.H. Pregnancy from invitro fertilization of human eggs after separation of motile spermatozoa by density gradient centrifugation. Fertil steril. 1986 45: 93-96

5. - LE LANNOU D., BLANCHARD Y. Nuclear maturity and morphology of human spermatozoa selected by Percoll density gradient centrifugation or swim-up procedure. J. Reprod. Fertil, 1988 84: 551-556 6. - LEVENTHOL A., MARGALISH E.J.,
SCHENKER J.C. Testing the fertilizing ability of motile spermatozoa separated by Percoll in patients with abnormal sperm analysis or sperm penetration. Int. J. Fertil., 1987 32: 302-305

RÉSUMÉ : La centrifugation sur gradient de Percoll discontinu est une technique de préparation des spermatozoïdes qui a permis d'améliorer les succès de la fécondation in-vitro. Dans ce travail, nous avons analysé les mouvements des spermatozoïdes séparés dans différentes fractions de Percoll après 4 heures d'incubation à $37^{\circ} \mathrm{C}$ et sous $5 \%$ de $\mathrm{CO} 2$. Les mesures sont faites à l'aide de la vidéomicrographie assistée par ordinateur (Cell Soft). Il ressort de cette étude que plus la concentration en Percoll augmente, plus la vitesse curvilinéaire, la linéarité et l'amplitude de déplacement latéral de la tête des spermatozoïdes augmentent. Les fractions $100 \%$ et 90 $\%$ de Percoll permettent d'obtenir des spermatozoïdes avec de très bonnes caractéristiques des mouvements indiquant l'usage de cette technique en procréation médicalement assistée. Mots clés : spermatozoïdes humains, Percoll, mobilité, vidéomicrographie. Andrologie, 1991, 1 : 9 -10 